EPJ Web of Conferences 59, 01011 (2013)

DOI: $10.1051 /$ epjconf/20135901011

(C) Owned by the authors, published by EDP Sciences, 2013

\title{
Experiments on extreme states of matter towards HIF at FAIR
}

\author{
Boris Sharkov ${ }^{1, a}$ and Dmitry Varentsov² \\ ${ }^{1}$ FAIR GmbH, Planckstrasse 1, 64291 Darmstadt, Germany \\ 2 GSI Helmholtzzentrum für Schwerionenforshung GmbH, Planckstrasse 1, \\ 64291 Darmstadt, Germany
}

\begin{abstract}
The Facility for Antiproton and Ion Research in Europe (FAIR) will provide worldwide unique accelerator and experimental facilities allowing for a large variety of unprecedented frontier research in extreme state of matter physics and applied science. Indeed, it is the largest basic research project on the roadmap of the European Strategy Forum of Research Infrastructures (ESFRI), and it is cornerstone of the European Research Area. FAIR offers to scientists from the whole world an abundance of outstanding research opportunities, broader in scope than any other contemporary large-scale facility worldwide. More than 2500 scientists are involved in setting up and exploiting the FAIR facility. They will push the frontiers of our knowledge in plasma, nuclear, atomic, hadron and applied physics far ahead, with important implications also for other fields in science such as cosmology, astro and particle physics, and technology. It includes 14 initial experiments, which form the four scientific pillars of FAIR. The main thrust of intense heavy ion and laser beam-matter interaction research focuses on the structure and evolution of extreme state of matter on both a microscopic and on a cosmic scale.
\end{abstract}

\section{INTERNATIONAL FAIR}

Construction of a new accelerator facility called FAIR (Facility for Antiproton and Ion Research) was started in 2010 as an international research project in Darmstadt, Germany. This new accelerator complex will consist of two powerful heavy ion synchrotrons and a number of storage rings and experimental facilities for various research projects $[1,2]$. The centrepiece of the accelerator assembly will be a $100 \mathrm{Tm}$ superconducting heavy ion synchrotron SIS-100 (see Fig. 1). FAIR will provide compressed beam pulses with an intensity that exceeds the current beam intensities by two orders of magnitude. This will extend the available beam deposition power from the current level of $10 \mathrm{GW} / \mathrm{g}$ by at least two orders of magnitude up to $3 \mathrm{TW} / \mathrm{g}$. Many aspects of high power beam physics associated with inertial confinement fusion driven by intense heavy ion beams will be addressed there, even though this facility will not provide enough beam power to ignite a fusion pellet [3]. Due to the unique feature of the energy deposition of heavy ions in dense matter-volume character of heating-it is possible to generate extreme states of matter that cannot be accessed with other drivers. This will open up the possibility to explore the thermo-physical and transport properties of high energy density (HED) matter in a regime that is very difficult to access using the traditional methods of shock compression [4].

\section{HED PHYSICS WITH INTENSE HEAVY ION BEAMS AT FAIR}

Recent progress and the expected future developments [5-7] in the technology of high quality, strongly bunched, well focused intense heavy ion beams have provided a very efficient tool to research HED physics in the laboratory.

\footnotetext{
ae-mail: boris.sharkov@fair-center.eu
}

This is an Open Access article distributed under the terms of the Creative Commons Attribution License 2.0, which permits unrestricted use, distribution, and reproduction in any medium, provided the original work is properly cited. 


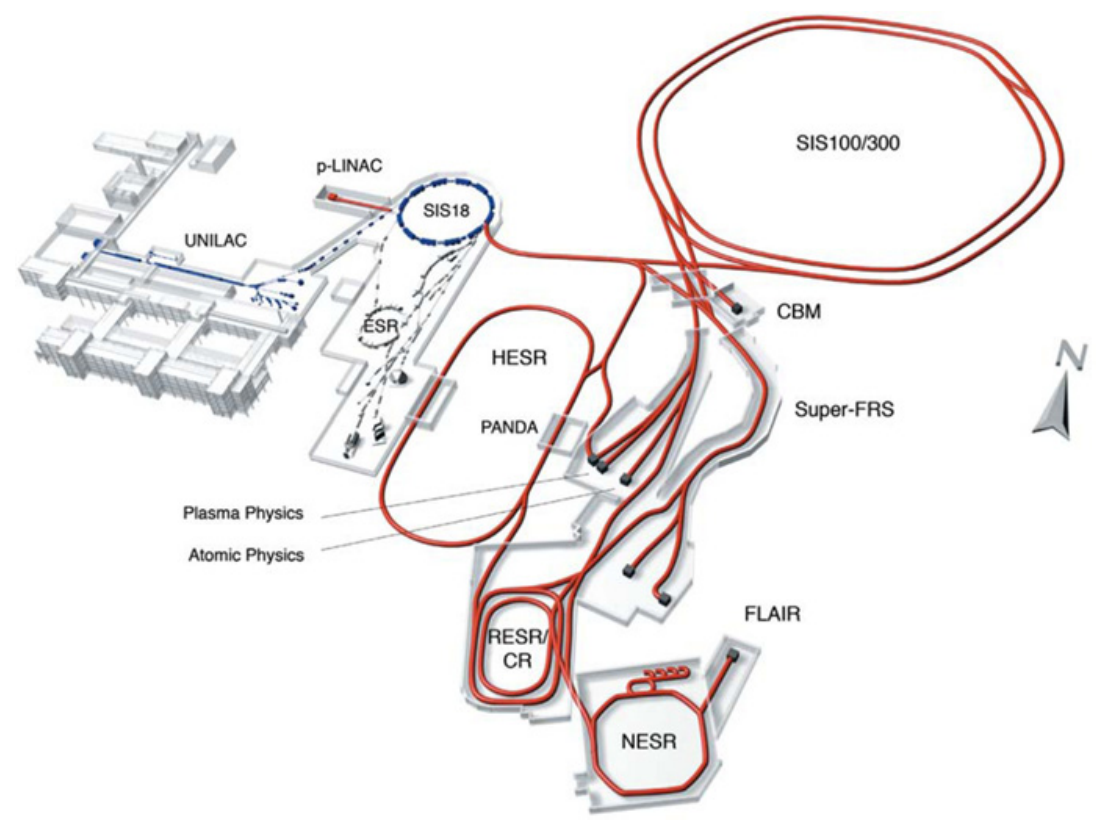

Figure 1. The FAIR facility.

In case of shock compression, matter is irreversibly heated and this generates states of high pressure, high entropy in the sample material. Intense particle beams, on the other hand, deposit their energy over extended volumes of matter and are thus capable of inducing these exotic states in the target material directly, without generation of shocks. The main advantages of using intense beams include high repetition rate of the driver, high beam - target coupling efficiency and rather large samples of HED matter with fairly uniform physical conditions (no sharp gradients).

Due to these reasons, a dedicated experimental program on HED physics has been proposed for FAIR [2], which is going to be one of the most powerful accelerators in the world with respect to the beam power and quality. Extensive experimental and theoretical work has been carried out over the past years to assess the potential of intense heavy ion beams to research this important field. This work has shown that one will be able to perform different types of experiments on HED physics at FAIR as described below.

\subsection{Studies on equation of state of HED matter}

An intense focused heavy ion beam heats large-volume targets uniformly and quasi-isochorically, thereby generating states of high pressure and high entropy. The heated material can then expand isentropically and depending on the deposited energy, will reach different physical states of HED matter. These include an expanded hot liquid, the region near the critical point, two-phase liquid-gas region, strongly coupled (non-ideal) plasmas and warm dense matter (WDM) regions. Such ion-beam generated samples can be used to study the Equation of State (EOS) properties of different phases of HED matter. This scheme is named HIHEX (Heavy Ion Heating and Expansion) [4, 8] and is an important part of the experimental program at FAIR. It is to be noted that some of the above HED states are either very difficult to achieve or are inaccessible using the traditional methods by shock in the limited region of the principal and porous Hugoniots (shock adiabats).

The thermodynamic path followed by the material in a HIHEX experiment can be accessed by depositing the corresponding amount of the specific energy and the subsequent expansion of the heated 
material. Therefore, using this technique, one will be able to study a wide region of the HED phase diagram of all the materials of interest at FAIR.

\subsection{Planetary physics research at FAIR}

Theoretical work has shown that an intense heavy ion beam can be efficiently employed to isentropically implode a sample material like hydrogen or water that will generate physical conditions which are expected to exist in the interiors of the giant planets in our solar systems as well as in exoplanets [9-12]. An experiment based on this scheme, named LAPLAS (Laboratory Planetary Sciences) has been proposed to study planetary physics at FAIR [9].

In a LAPLAS experiment, the target consists of a cylinder of the sample material (e.g., frozen hydrogen or water) that is surrounded by a thick shell of a heavy material, like gold or lead. One face of the target is irradiated with an intense heavy ion beam that has an annular (ring-shaped) focal spot, and the target length is less than the range of the driver ions so that the energy deposition in the longitudinal direction is uniform. Such a hollow heavy ion beam can be generated using a RF fast beam rotator (wobbler) system [13]. It is assumed that the inner radius of the annulus is larger that the radius of the sample material which is a necessary condition to avoid direct heating of the sample by the ion beam. A layer of cold material from the outer shell known as "pusher" or "payload", is thus created between the sample material and the beam-heated region. The payload plays an important role in placing the compression on the desired adiabat.

This scheme generates a low-entropy compression that leads to a very high material density with a relatively low temperature. Simulations have shown that using the parameters of the SIS-100 beam at FAIR, one would achieve hydrogen density of $1-3 \mathrm{~g} / \mathrm{cm}^{3}$, pressure of 3-30 Mbar and temperature on the order of ten thousand Kelvin. These are the predicted physical conditions in the interior of Jupiter.

\subsection{Relevance to heavy ion IFE}

Cylindrical implosion suggested in LAPLAS experiments is relevant to the Heavy Ion Fusion Energy concept, based on the high-energy ion beams drive of a cylindrical target developed in [14, 15].

Direct drive of cylindrical DT-target by intense ion beam in fast ignition mode and wetted liquid wall reactor chamber design with lead-lithium blanket are the basic features of this concept. This approach is characterized by a simple driver-reactor chamber interface and a moderate value of demanded thermonuclear gain.

In accordance with suggested scenario, because of relatively long ranges $\left(5-10 \mathrm{~g} / \mathrm{cm}^{2}\right)$ of $\sim 100 \mathrm{GeV}$ heavy ions, it is natural to use a cylindrical rather than a spherical target. Relatively low efficiency of the cylindrical implosion in comparison with spherical one is partly compensated by using the direct drive. High degree of azimuthal uniformity of the ion energy deposition needed for direct drive is ensured by fast rotation of the compression beam around the target axis, so that the beam heats a ring area of the lead shell outside the DT/lead interface. As it has been shown in $[15,16]$, the revolution of the beam at frequency of $1 \mathrm{GHz}$ during the main pulse should be sufficient to reach the needed radial convergence.

The target consists of a uniform lead tube shell filled with DT fuel ice. The end surface of the shell is axially irradiated by the hollow ion beam formed by a high frequency rotation of the pointed beam. Target compression and burn is computed by the DEIRA-4 one-dimensional three-temperature Lagrangian code [16] which includes diffusion of radiation in the one-frequency-group approximation and the fast fusion ions, nuclear reaction kinetics, heating rates by neutrons and by fast heavy ions. The equation of state approximates realistic properties of materials in the region of strong coupling.

The length of the main RF linac is of the order of $10 \mathrm{~km}$. In the case of most complicated target operation version - fast ignition scenario - the driver requires certain complications [17]. In the main linac the final ion energy ends up with $\sim 500 \mathrm{AMeV}$. The pre-compression of the target is accomplished by the temporally profiled beam over duration of $75 \mathrm{~ns}$ with a maximum current of $1.6 \mathrm{kA}$. 
Repetition rate of the driver is taken as $8 \mathrm{~Hz}$, which provides two shots per second in each of four reactor chambers. The nominal driver efficiency is equal to 0.25 .

The general design of the reactor chamber is given in $[17,18]$. The chamber consists of the two cylindrical sections: the upper smaller section in which the target explosion takes place and the lower section in which sprayed jets of coolant are injected for condensation of evaporated liquid. The diameters of the sections are $8 \mathrm{~m}$ and $16 \mathrm{~m}$, respectively.

The chamber first wall and the reactor blanket are of conventional design: the LiPb liquid film is formed at $\mathrm{SiC}$ porous wall. Taking into account of the driver efficiency, the target gain and the blanket multiplication for the fusion power of $1500 \mathrm{MW}$ the net efficiency of the plant of 0.373 and net power per one reactor of $626 \mathrm{MW}$ has been specified.

A number of heavy ion IFE related issues will be addressed as soon as FAIR facility will be commissioned. Those are both accelerator-driver issues like generation of high phase space density heavy ion beams, beam transport and final focusing, generation of circular beams and homogeneous irradiation of a cylindrical target. Target physics issues to be explored include quasi-adiabatic cylindrical compression, isochoric heating of matter and EOS of HED matter.

\section{REQUIREMENTS TO PERFORMANCE OF EXPERIMENTAL CAMPAIGN}

The properties of matter under conditions of high density and pressure determine the hydrodynamic response of a fusion pellet to an intense pulse of ion or laser radiation. Therefore it is necessary to measure these properties experimentally. Especially the regime of high density at moderate temperature which is often called Warm Dense Matter (WDM) is of special interest since during the irradiation process the fusion target material passes through this regime. Therefore the determination of the equation of state is a topic of intense research effort experimentally with intense ion beams resulting in challenging requirements for beam and target diagnostics in HED physics experiments at FAIR.

The FAIR facility will be capable of generating highly compressed (50-70 ns) pulses of heavy elements up to ${ }^{238} \mathrm{U}$ with intensity in the range $5 \cdot 10^{10}-5 \cdot 10^{11}$ ions per pulse. These beams will be focused down to the spot of $\sim 1 \mathrm{~mm}$ in diameter. Due to the use of special rotation system [13, 16], an annular focal spot might be generated for the LAPLAS experiments with cylindrical targets. Precise measurements of the beam energy, intensity and of the intensity distribution in the focal spot [19] will provide data for specific energy deposition.

Determination of the target density distribution is a great challenge for both HIHEX and LAPLAS experiments: the expected target areal density is up to $20 \mathrm{~g} / \mathrm{cm}^{2}$ ( $\mathrm{Pb}, \mathrm{Au}$, etc.) whereas the density distribution has to be measured with rather high spatial $(10 \mu \mathrm{m})$ and temporal $(10 \mathrm{~ns})$ resolution with the density reconstruction accuracy at sub-percent level. This problem can be uniquely solved by high energy proton microscopy (HEPM) technique [21-23]. For this purpose, an additional perpendicular proton beam line from the existing SIS-18 synchrotron will constructed for the HED physics experiments at FAIR providing a proton microscopy capability with $4.5 \mathrm{GeV}$ intense proton beams [2].

GSI-Darmstadt is also the first accelerator laboratory worldwide where in addition to a powerful and intense heavy ion beam a high-energy laser beam [24] is available for experiments using laser and particle beams simultaneously. A high energy PW-class laser will also be available at the FAIR facility for target diagnostics purposes [2].

\section{CURRENT ACTIVITIES ON HED PHYSICS WITH INTENSE HIGH ENERGY ION BEAMS}

The "High-Temperature" (HHT) was specifically designed for WDM experiments with intense heavy ion beams. This area is equipped with a strong final focusing system, diagnostics for intense, submicrosecond ion pulses and a vacuum target chamber. A comprehensive experimental setup for WDM 
experiments has been developed and built up throughout the past decade [19, 20, 25-31]. The main aims of the WDM experiments performed at the HHT area of GSI were commissioning of recently developed diagnostic instruments and methods for the future HED physics experiments at FAIR; tests of different beam-target configurations for EOS studies; optimization of transport, focusing and diagnostics of intense heavy ion beams; obtaining new data on thermo-physical properties and hydrodynamic response of various materials in HED states near boiling curve, two-phase liquid-gas and the critical point regions. In particular, HED properties of lead, tin, copper, aluminum, tungsten, tantalum, and sapphire have been studied using the "plane-HIHEX" design concept [19, 29, 31].

In the performed experiments, electron-cooled beams of ${ }^{238} \mathrm{U}^{73+}$ ions delivered by the SIS-18 synchrotron with initial ion energy of 200-400 AMeV have been used. The intense, up to 4-5 $10^{9}$ ions/bunch, ion pulses have been longitudinally compressed down to 100-130 ns (FWHM) employing fast bunch rotation technique and focused at the target to a spot of $\sim 300 \mu \mathrm{m}$ FWHM using a conventional quadrupole triplet. Such a beam can generate in solid metals WDM states characterized by specific energies of a few $\mathrm{kJ} / \mathrm{g}$, temperatures up to $2 \mathrm{eV}$ and pressures in the multi-kbar range.

Recently an international project was started for the development of a new proton microscopy facility in order to advance heterogeneous material and warm dense matter studies. This facility called PRIOR (Proton Microscope for FAIR) [23, 32, 33] will provide a significant step forward in both spatial and temporal resolution. Its installation at GSI and commissioning in dynamic experiments with the $4.5 \mathrm{GeV}$ proton beam from the SIS-18 synchrotron is planned for 2013. This worldwide unique proton microscope facility will provide at GSI and at FAIR a capability for high-precision experiments in plasma physics, high energy density physics, materials research and biophysics. The technical specifications of the PRIOR facility correspond to the requirements for the HED physics experiments that will be performed by the HEDgeHOB collaboration at FAIR [2, 34].

\section{SUMMARY}

The Facility for Antiproton and Ion Research in Europe, FAIR, will provide worldwide unique accelerator and experimental facilities allowing for research into extreme state of matter physics. Extensive theoretical work has been carried out over the past years to assess the potential of the FAIR ion beams to research this important field. This work has shown that one will be able to perform different types of experiments on HED physics at FAIR. During the past years, significant progress has been achieved in experimental investigation on heavy-ion beam generated high-energy-density (HED) states in matter (warm dense matter, WDM) by using the GSI-heavy ion accelerator, the most powerful and versatile heavy ion accelerator worldwide.

The authors would like to thank D.H.H. Hoffmann, A.R. Piriz and N.A. Tahir for helpful discussions and their invaluable contributions to the subject of this work.

\section{References}

[1] C. Sturm, H. Stocker, B. Sharkov, Nucl. Phys. A 834, 682 (2010)

[2] FAIR Baseline Technical Report (ed. H.H. Gutbrod, GSI, Darmstadt 2006)

[3] D.H.H. Hoffmann et al., Laser and Part. Beams 23, 47 (2005)

[4] D.H.H. Hoffmann, V.E. Fortov et al., Phys. Plasmas 9, 3651 (2002)

[5] W.F. Henning, Nucl. Inst. and Meth. B 214, 211 (2004)

[6] A. Friedman et al., Nucl. Instr. and Meth. A 606, 6 (2009)

[7] J.J. Barnard et al., Nucl. Instr. and Meth. A 606, 134 (2009) 
[8] N.A. Tahir et al., Nucl. Inst.and Meth. A 606, 128 (2009)

[9] N.A. Tahir et al., Phys. Rev. E 63, 016402 (2001)

[10] N.A. Tahir et al., Astrophys. Space Sci. 322, 179 (2009)

[11] A.R. Piriz et al., Phys. Rev. E 66, 056403 (2002)

[12] A. Grinenko, D.O. Gericke, D. Varentsov, Laser and Part. Beams 27, 595 (2009)

[13] S. Minaev et al., Nucl. Instr. Meth. A 620, 99 (2010)

[14] B.Yu. Sharkov, N.N. Alexeev, M.M. Basko et al., Nuclear Fusion 45, 291 (2005)

[15] B.Yu. Sharkov, Nucl. Inst. and Meth. A 577, 14 (2007)

[16] N.N. Alekseev, M.M. Basko, E.A. Zabrodina et al., Atomic Energy 97, 632 (2004)

[17] Yu.N. Orlov, M.M. Basko et al. Nuclear Fusion 45, 531 (2005)

[18] S.A. Medin et al., Nucl. Inst. and Meth. A 544, 300 (2005)

[19] D. Varentsov, A. Fertman, V.I. Turtikov et al., Contrib. Plasma Phys. 48, 586 (2008)

[20] D. Varentsov, V.Ya. Ternovoi, M. Kulish et al., Nucl. Inst. and Meth. A 577, 262 (2007)

[21] N.S.P. King et al., Nucl. Instr. and Meth. A 424, 84 (1999)

[22] A.A. Golubev et al., Tech. Phys. Lett. 36, 177 (2010)

[23] High Energy Proton Microscopy workshops series: http://lansce.lanl.gov/ proton\%20microscopy; http://www.ficp.ac.ru/hepm2010; http://www-aix.gsi.de/ conferences/HEPM2009 (2009-2011)

[24] D.H.H. Hoffmann, A. Blazevic, S. Korostiy, et al., Nucl. Inst. and Meth. A 577, 8 (2007)

[25] U. Neuner et al., Phys. Rev. Lett. 85, 4518 (2000)

[26] D. Varentsov et al, Europhys. Lett. 64, 57 (2003)

[27] C. Constantin et al., Rev. Sci. Instr. 75, 1268 (2004)

[28] S. Udrea et al., J. Phys. A: Math. Gen. 39, 4743 (2006)

[29] P. Ni et al., J. Phys. IV 133, 977 (2006)

[30] A. Ulrich et al., Phys. Rev. Lett. 97, 153901 (2006)

[31] D. Nikolaev et al., High Temp. — High Press. 40, 281 (2011)

[32] F.E. Merrill et al., AIP Conf. Proc. 1195, 667 (2009)

[33] PRIOR Technical Design Report, GSI-JIHT-IPCP-ITEP-LANL (2009)

[34] HEDgeHOB (High Energy Density Matter Generated by Heavy Ion Beams) collaboration at FAIR: http://www.fair-center.eu/fair-users/experiments.html; http:// hedgehob.physik.tu-darmstadt.de/ 